\title{
Exploring Interdisciplinary Relationships Among King Abdulaziz University Departments via ResearchGate: Network Analysis and Visualizations
}

\author{
Hanan M. Baaqeel ${ }^{1}$, Sara F. Aloufi ${ }^{1} \&$ Tariq Elyas $^{2}$ \\ ${ }^{1}$ Department of Statistics, KAU—King Abdulaziz University, Jeddah, Saudi Arabia \\ ${ }^{2}$ Department of European Languages and Literature, KAU_King Abdulaziz University, Jeddah, Saudi Arabia \\ Correspondence: Tariq Elyas, Department of European Languages and Literature, KAU_King Abdulaziz University, \\ Jeddah, Saudi Arabia. E-mail: telyas@kau.edu.sa
}

Received: August 20, 2020

Accepted: September 21, 2020

Online Published: September 26, 2020

doi:10.5430/jms.v11n3p55

URL: https://doi.org/10.5430/jms.v11n3p55

\begin{abstract}
Because all disciplines are connected, interdisciplinary studies are one of the most significant discussions in the education sector. It involves the merging of two or more academic disciplines into one activity. The aim of this research paper is to explore the relationship of interdisciplinary research and network among all departments at King Abdulaziz University (KAU) in ResearchGate (RG) by using the statistical network analysis of undirected social networks. In our academic network, the departments of the university represent the vertices and their academic relationships. We will detect the communities between the departments in RG network by using statistical analysis of the network for each community. Finally, we will compare the academic social network at KAU to some random graph models, and investigate some random graph characteristics, such as power-law, small-world, and scale-free models. In our research, we found that the Department of Chemistry has the highest degree for the academic social network at KAU in RG, and the highest eigenvector centrality as well. In terms of vertex centrality, the Department of Electrical and Computer Engineering has the highest value in closeness and betweenness centrality. Also, we found that the most two connected departments are the Department of Computer Science and Department of Physics through the edge weight equals 248 . By using community detection, we found there are seven communities. We conclude that the degree distribution of the academic social network of KAU in RG is different from the degree distribution of random graph models, but it is slightly close to small world model. This study, in turn, can participate to achieve one of the goals of Vision 2030 by shedding some light into how to improve research networks in the education sector and research among Saudi universities.
\end{abstract}

Keywords: community deduction, descriptive network analysis, random graph models, interdisciplinary research, KAU, ResearchGate, research network

\section{Introduction}

A social network consists of a set of vertices connected by edges where each edge connects the two vertices, where the edges can have a direction. Analysis of social networks is similar to a traditional statistical analysis in its steps but differs in its tools. First, the social networks are first studied through the network representation. The descriptive analysis of the network is the second step. Then, the statistical analysis of the social network is done by modeling and examining of the model. In our research paper, we are interested in the descriptive analysis of undirected academic social networks basic description, characteristics vertex and edges, sub- groups, and graph partitioning. The social network used in this research is the academic social network among all the departments at KAU within the RG platform.

\section{Literature Review}

Interdisciplinary research has become more common in scientific research and defined as the integration of disciplines within a research field. A study by (Hu \& Zhang, 2017) conducted on the social network by taking the data from Web of Science used statistical analysis of the network. The results were irregular disciplinary distribution and that the most effective disciplines are Computer Science, Engineering, Business, and Economics. However, Mislove, Marcon, Gummadi, Druschel, \& Bhattacharjee (2007) used the other important online social networks: 
Flickr, YouTube, LiveJournal, and Orkut. The results achieved characteristics power-law, small-world, and scale-free. They observed that there is an inverse relationship between the node degree in the cluster and clustering coefficient. Whereas, Elsayed (2016) found that RG is the most widely used platform by researchers to publish their research. She conducted his t survey on six Arab universities (KAU participated in the survey). She has found that $\mathrm{RG}$ is the most widely used by researchers to publish their research. In contrast, a study by Kraker and Lex, (2015) states that the RG Score given to researchers should not be considered at present because it suffers from limitations in several places: non-transparent, non-repeatable, affected by the individual assessment of researchers, and change in result cannot be structured.

There is a relatively small body of literature that is concerned with modelling, a study by Srivastava, Anuradha, and Gupta (2014) pointed that the analysis of the social network provides future social networks through modeling. The existing literature (De Andrade \& R`ego, 2018), on social network is extensive and focuses particularly on the complexities of the social network in four ways: unweighted, edge-weighted, two methods for using both edge-weights, and nodes attributes.

There are relatively few historical studies in scientific research network, one of them (Pandia \& Bihari, 2015). They studied the amount of cooperation between the researchers and the amount of the researcher's performance in this network. They found every measure of centrality given different to each researcher. Moreover, a large and growing body of literature has investigated different online activities. The research by Hassan et al., (2017) examined the online activity of scholarly publications, such as news sites, social media platforms, blogs, video sites, and reference management tools. The researchers pointed out that the social media activities, across disciplines, increase in the presence of Altmetric.com data in Scopus database, rapidly. They used a zero truncated negative binomial model to determine the association of various factors with citations. As a result, they conclude that Altmetric indices could be a valuable indicator to distinguish publications with high citation impact. Furthermore, a study by Pandia and Bihari (2015) conducted on the scientific research network to study the amount of cooperation among the researchers and the amount of the researcher's performance in this network and adopted in its analysis on the following measures: degree centrality, closeness centrality, betweenness centrality and eigenvector centrality. They found every measure of centrality given different researcher. For this research paper, a graph theory concepts, and statistical methods are used to analyze and visualize the academic social network. For more details see (Luke, 2015a) and (Kolaczyk \& Csa'rdi, 2014).

\section{Research Objectives}

1. Finding a general summary describing the academic social network and discover the central departments and finding the strong relationships between departments.

2. Discovering communities in the academic network and find the characteristics of each community.

3. Comparing random graph models to the academic social network.

In 2016, Prince Mohammed Bin Salman Al- Saud- Crown Prince and Chairman of the Council of Economic and development Affairs stated a new plan known as Vision 2030. Improving the education sector is one of the goals of the vision. One of the ambitious vision's goals is to work towards having at least five Saudi universities among the top 200 universities in international rankings (Mosaad, 2016).

\section{Application}

\subsection{Data Collection}

This paper aims on analyzing the academic social network of KAU in ResearchGate where the departments of the university represent the vertices and relations between them represent the edges. In the time of this research, the account KAU in the RG had 144 departments, so we have 144 vertices. Each department contains several registered account members. The data of the edges were collected by looking at each member of the department who fall under the 144 departments at KAU. This process was repeated to all members in each department. Other data were added which are the characteristics of each department collected from the account of each department. The attributes collected are the following: 1) number of members, 2) publications, 3) authors, 4) $H$-index, 5) $H$-index self and 6) RG score. One important observation is that the academic network changes every day. We must mention that the period in which these data were collected was from 10 November to 30 November 2017. The software used in this research are SPSS to insert the academic network data, and R to analyze academic network data. The packages used "igraph", "network", "statnet" and "linkcomm". Finally, we used Gephi for visualization of the academic network. For the academic network, an index was assigned to each department for abbreviation which is shown in the Appendix. 


\subsection{Descriptive Statistics}

We summarized the academic social network among the departments at KAU via RG and the descriptive statistics of all attributes are illustrated in the Table 1.

Table 1. The highest department in each attribute for the academic social network of KAU in RG

\begin{tabular}{lllll}
\hline Attributes & Index & Department Name & Value & Mean \\
\hline Number of members & 1 & Computer Science & 278 & 29 \\
\hline Publications & 3 & Chemistry & 271 & 19 \\
\hline Authors & 3 & Chemistry & 450 & 36 \\
\hline$h$-index & 4 & Biochemistry & 34 & 9.36 \\
\hline$h$-index self & 43 & Medical Education & 30 & 8.63 \\
\hline RG.score & 2 & Biological & 124.53 & 22 \\
\hline
\end{tabular}

The attribute number of members represents the number of members in each department with the mean of 29 . The department that received the highest number of members with a value of 278 members was the Department of Computer Science, and the lowest number of members was zero which was found in the following departments: Home Economics, Department of Emergency Medical Services, Department of Hospitality and Hotel Management, Faculty of Medical Sciences, Geological Hazards Research Unit and Tourism Institute (now Faculty of Tourism). The attribute publications represent the number of publications in each department with mean of 19. The department that received the highest number of publications with a value of 271 publications was Department of Chemistry. The attribute Authors represents the number of authors in each department with the mean of 36 . The department that received the highest number of authors with a value of 450 authors was Department of Chemistry. The attribute $h$.index represents the top $h$ cited research for each top department member with the mean of 9.36. The department that received the highest $h$.index with a value equal 34 was Department of Biochemistry. The attribute $h$.index.self represents the excluding self-citations for each top department member with the mean of 8.63 and the department that received the highest $h$.index.self with a value equals 34 was Department of Medical Education.

The attribute $R G$ score whereas the RG score takes all of the scholar's research and turns it into a source of reputation. In this study, we took RG score only for the top member in each department. We found that the mean equals 22 and the department that received the highest RG score with a value equal 124.53 was Department of Biological Science.

\subsection{Basic Description}

The academic social network structure is illustrated in Figure 1 and the basic description of the network is shown in Table 2. 


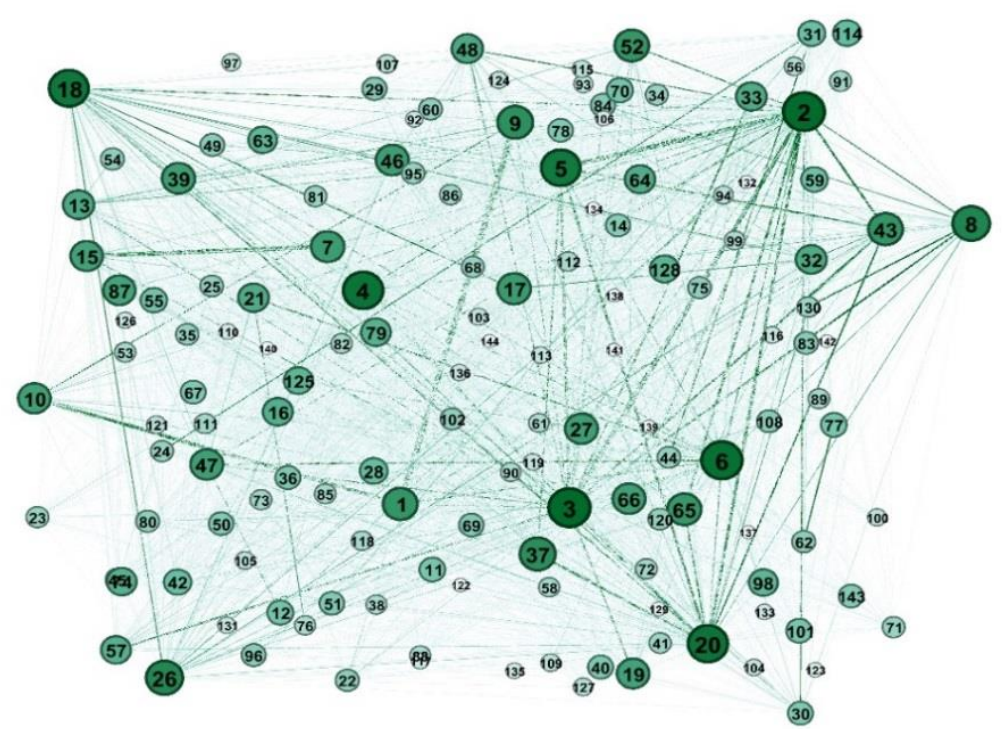

Figure 1. The academic social network of KAU in RG

Table 2. Basic description for the academic social network of KAU in RG

\begin{tabular}{lllll}
\hline Size & Density & Components & Diameter & Clustering coefficient \\
\hline 144 & 0.35 & 5 & 5 & 0.58 \\
\hline
\end{tabular}

1. The size is the number of departments in the academic social network of KAU in RG with 144 departments.

2. Density is the proportion of observed connection in a network to the maximum number of possible connections which is equal 0.35 which expressed a moderate level of connection.

3. Components which means the network to how much subgroup is divided which equals 5 .

4. Diameter is the longest way to go from department $\mathrm{X}$ to department $\mathrm{Y}$ which equals 5 steps.

5. Clustering coefficient is a ratio of closed triangles to closed and open triangles which equals 0.58 which was a strong level of clustering.

The vertex characteristics are shown in Table 3.

Table 3. The highest department in each vertex characteristic for the academic social network of KAU in RG

\begin{tabular}{|c|c|c|}
\hline Degree & Eigenvector & Betweenness \\
\hline Chemistry & & Electrical \& Computer Engineering \\
\hline
\end{tabular}

Characteristics of Edges was the highest weight of the edge which expresses the interdisciplinarity and the strongest relationship between Department of Computer Science and Department of Physics which equals to 248, and the ten strongest relations between departments can be in the Table 4.

Table 4. The ten strongest relations between departments by weight of edges

\begin{tabular}{lll}
\hline Department A & Department B & Weight \\
\hline Computer Science.1 & Physics.9 & 248 \\
\hline Biological Science.2 & Mathematics.5 & 199 \\
\hline Biological Science.2 & Chemistry.3 & 184 \\
\hline English Language Institute.7 & Nursing.15 & 162 \\
\hline
\end{tabular}




\begin{tabular}{lll}
\hline Computer Science.1 & Information Technology.10 & 123 \\
\hline Biological Science. 2 & Food and Nutrition.20 & 115 \\
\hline Chemistry.3 & Mathematics.5 & 107 \\
\hline Biological Science.2 & Faculty of Computing and Information Technology.65 & 107 \\
\hline Mathematics.5 & Food and Nutrition.20 & 96 \\
\hline Food and Nutrition.20 & Dentistry.37 & 94 \\
\hline
\end{tabular}

In Vertex Degree from the Figure 2, we can see many of the departments have low degrees and the minority have high degrees. In Figure 3, shows the academic social network of KAU in RG where the size of the vertex represents the degree of this department. The top three departments which received the highest degrees were the following: Department of Chemistry, Department of Biological Science and Department of Electrical and Computer Engineering. The least three departments which received the least degrees are the following: Home Economics, Department of Emergency Medical Services, Faculty of Medical Sciences and Tourism Institute (now Faculty of Tourism).

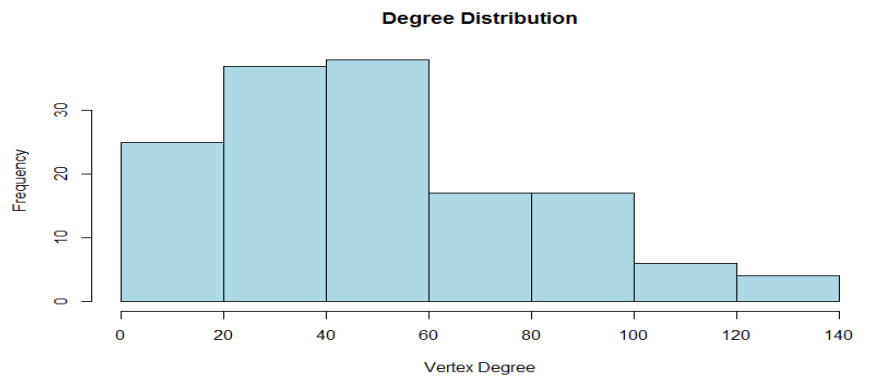

Figure 2. The original scale of degree distribution for the academic social network of KAU in RG

\subsubsection{Closeness Centrality}

The department with the highest closeness centrality is the closest one to all other departments which was the Department of Electrical and Computer Engineering in our investigation. Through Figure 3 we can see the closeness centrality for the academic social network of KAU departments in RG.

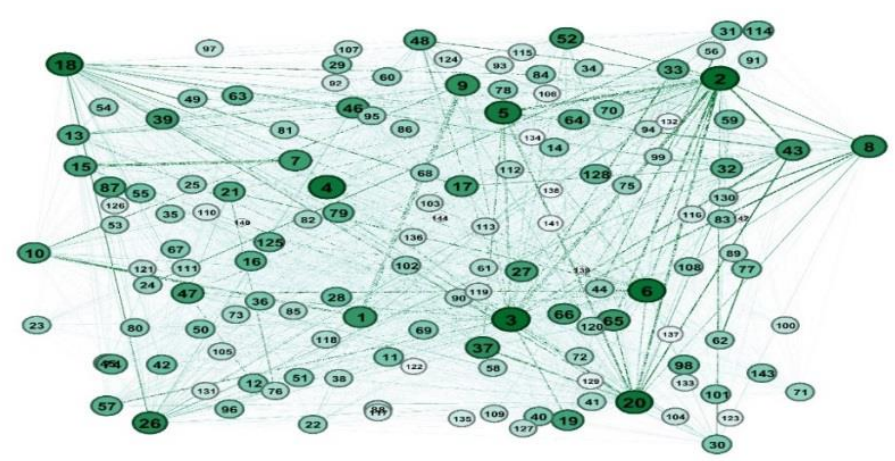

Figure 3. The closeness centrality for the academic social network of KAU in RG

\subsubsection{Betweenness Centrality}

The department with the highest betweenness centrality, and the bridge between other departments were as following: The Department of Electrical and Computer Engineering. Through Figure 4, we can see the betweenness centrality for the academic social network of KAU departments in RG. 


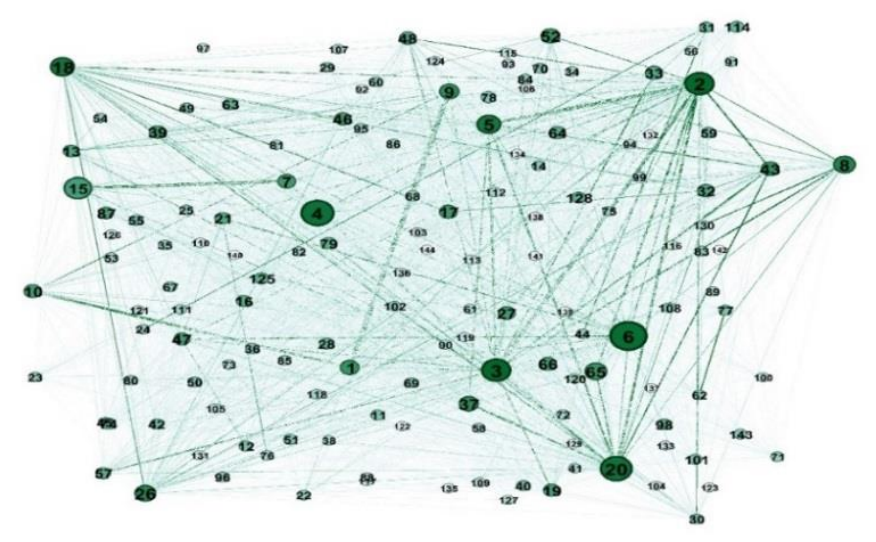

Figure 4. The betweenness centrality for the academic social network of KAU in RG

\subsubsection{Eigenvector Centrality}

The department with the highest eigenvector centrality is connected to many departments. The department with the highest eigenvector centrality was the Department of Chemistry. Through Figure 5 we can see the eigenvector centrality for the academic social network of KAU departments in RG.

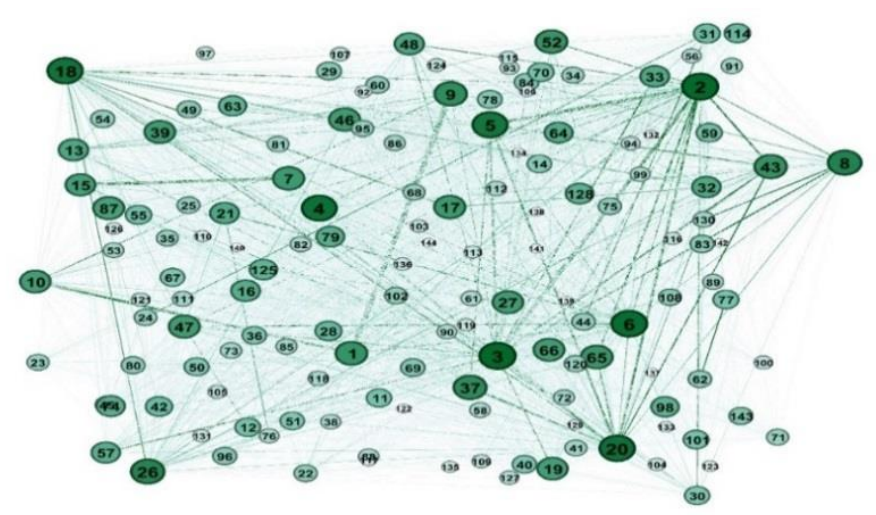

Figure 5. The eigenvector centrality for the academic social network of KAU in RG

\subsection{Community Detection}

In term of Social Cohesion, we are using the hierarchical clustering to divide them for the academic network of KAU in RG. We found seven clusters or communities as shown in Figure 6. It should be noted that the isolated departments of the network have been deleted and were the following departments: Department of Home Economics (139), Department of Emergency Medical Services (140), Faculty of Medical Sciences (142) and Tourism Institute (144). Modularity used to measure the strength of the hierarchical clustering to divide them for the academic network of KAU in RG were equal 0.15 which expresses a moderate level of modularity (Luke, 2015b). 


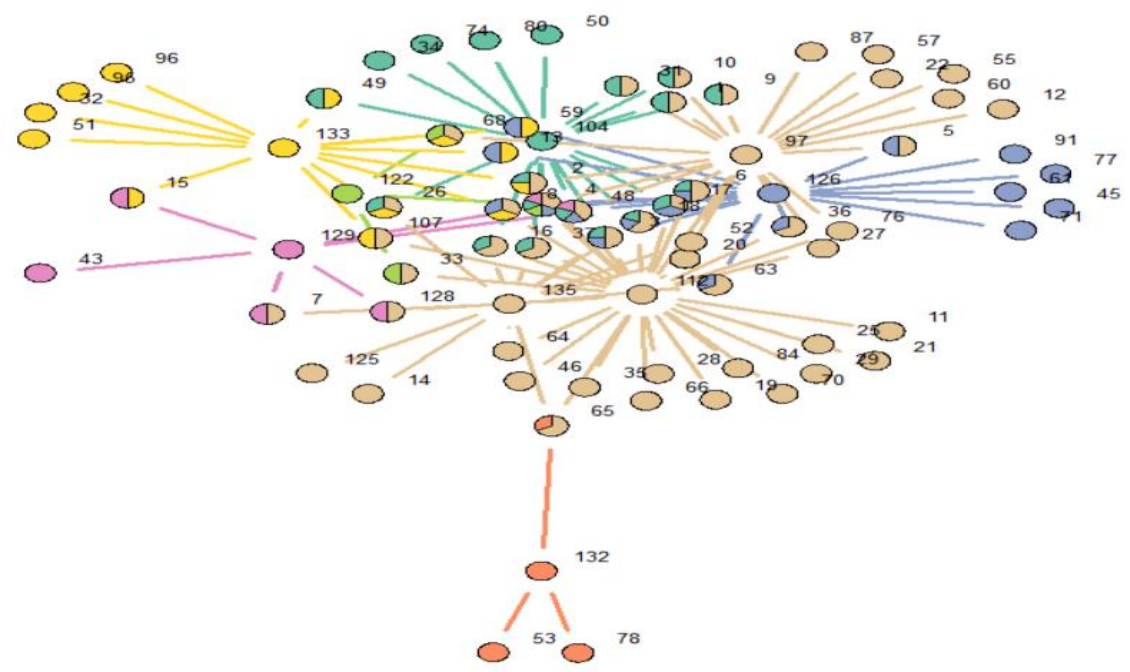

Figure 6. Hierarchical clustering for the academic social network of KAU in RG

\subsubsection{The First Cluster}

The departments of the first cluster (community) are illustrated in Table 5.

Table 5. Names of departments in the first cluster for the academic social network of KAU in RG

\begin{tabular}{llll}
\hline Index & Name of department & Index & Name of department \\
\hline 4 & Biochemistry & 48 & Management Information Systems \\
\hline 43 & Medical Education & 128 & Faculty of Sciences \\
\hline 15 & Nursing & 129 & Princess Al-Jawhara Center \\
\hline
\end{tabular}

\subsubsection{Basic Description}

1. The size of the first cluster is 7 departments.

2. Density is equal 1 which expresses a high level of connection.

3. Components are equal which means the network to how much subgroup is divide which equals 1.

4. Diameter is equal 1 step.

\subsubsection{The Second Cluster}

The departments of the second cluster (community) are illustrated in Table 6.

Table 6. Names of departments in the second cluster for the academic social network of KAU in RG

\begin{tabular}{llll}
\hline Index & Name of department & Index & Name of department \\
\hline 1 & Computer Science & 26 & Pediatrics \\
\hline 2 & Biological & 31 & Civil Engineering \\
\hline 3 & Chemistry & 34 & Microbiology \\
\hline 4 & Biochemistry & 37 & Dentistry \\
\hline 6 & Electrical \&Computer Engineering & 48 & Management Information \\
\hline 9 & Physics & 49 & Education Technology \\
\hline 10 & Information Technology & 50 & Health Services \\
\hline
\end{tabular}




\begin{tabular}{llll}
\hline 16 & Statistics & 74 & Radiology \\
\hline 17 & Chemical\& Materials Engineering & 80 & Production Eng. \\
\hline 18 & Internal Medicine & 104 & Natural Products \\
\hline
\end{tabular}

\subsubsection{Basic Description}

1. The size of the second cluster is 20 departments.

2. Density is equal 0.89 which expresses a high level of connection.

3. Components are equal which means the network to how much subgroup is divide which equals 1.

4. Diameter is equal 2 steps.

5. Clustering coefficient is 0.91 which is a very strong level of clustering. The highest eigenvector centrality in the second cluster was Department of Computer Science (1).

\subsubsection{The Third Cluster}

The departments of the third cluster (community) are illustrated in Table 7.

Table 7. Names of departments in the third cluster for the academic social network of KAU in RG

\begin{tabular}{llll}
\hline Index & Name of department & Index & Name of department \\
\hline 53 & Medical Technology & 78 & Hydrology \\
\hline 65 & Computing \& Information Technology & 132 & Applied Medical Sciences \\
\hline
\end{tabular}

\subsubsection{Basic Description}

1. The size of the third cluster is 4 departments.

2. Density is equal 0.83 Which expresses a high level of connection.

3. Components are equal which means the network to how much subgroup is divide which equals 1 .

4. Diameter is equal 2 steps.

5. Clustering coefficient is 0.75 which is a very strong level of clustering. The highest eigenvector centrality in the third cluster were Department of Hydrology Department (78) and Applied Medical Sciences Department (132).

\subsubsection{The Fourth Cluster}

The departments of the fourth cluster (community) are illustrated in Table 8.

Table 8. Names of departments in the fourth cluster for the academic social network of KAU in RG

\begin{tabular}{llll}
\hline Index & Name of department & Index & Name of department \\
\hline 4 & Biochemistry & 68 & Petroleum Geology \& Sedimentology \\
\hline 33 & Faculty of Medicine & 122 & Islamic Studies \\
\hline
\end{tabular}

\subsubsection{Basic Description}

1. The size of the fourth cluster is 4 departments.

2. Density is equal 1 which expresses a high level of connection.

3. Components are equal which means the network to how much subgroup is divide which equals 1 .

4. Diameter is equal 1 step.

5. Clustering coefficient is 1 which is a very strong level of clustering. The highest eigenvector centrality in the fourth cluster was the Department of Bio- chemistry (4).

\subsubsection{The Fifth Cluster}

The departments of the fifth cluster (community) are illustrated in Table 9 
Table 9. Names of departments in the fifth cluster for the academic social network of KAU in RG

\begin{tabular}{llll}
\hline Index & Name of department & Index & Name of department \\
\hline 3 & Chemistry & 45 & Environmental Sciences \\
\hline 4 & Biochemistry & 48 & Management Information Systems \\
\hline 5 & Mathematics & 59 & Diagnostic Radiology \\
\hline 6 & Electrical and Computer Engineering & 61 & Physiology \\
\hline 8 & Information Systems & 63 & Center of Excellence \\
\hline 13 & Business Administration & 71 & Marine Physics \\
\hline 17 & Chemical and Materials Engineering & 77 & Clinical Pharmacy \\
\hline 18 & Internal Medicine & 91 & Human Resources Management \\
\hline 36 & Nuclear Engineering & 126 & Marine Sciences \\
\hline
\end{tabular}

\subsubsection{Basic Description}

1. The size of the fifth cluster is 18 departments.

2. Density is equal 0.80 which expresses a high level of connection.

3. Components are equal which means the network to how much subgroup is divide which equals 1.

4. Diameter is equal 2 steps.

5. 5. Clustering coefficient is 0.84 which is a very strong level of clustering. The highest eigenvector centrality in the fifth cluster was the Department of Chemistry (3).

\subsubsection{The Sixth Cluster}

The departments of the sixth cluster (community) are illustrated in Table 10.

Table 10. Names of departments in the sixth cluster for the academic social network of KAU in RG

\begin{tabular}{|c|c|c|c|}
\hline Index & Name of department & Index & Name of department \\
\hline 2 & Biological & 51 & Anatomy \\
\hline$\overline{8}$ & Information Systems & 59 & Diagnostic Radiology \\
\hline$\overline{13}$ & Business Administration & 68 & Petroleum Geology and Sedimentology \\
\hline 15 & Nursing & 95 & Law \\
\hline 26 & Pediatrics & 96 & Islamic Economics Institute \\
\hline 32 & Marine Engineering & 107 & Structural Geology and Remote Sensing \\
\hline$\overline{49}$ & Education Technology & 133 & Economics and Administration \\
\hline
\end{tabular}

\subsubsection{Basic Description}

1. The size of the sixth cluster is 13 departments.

2. Density is equal 0.67 which expresses a high level of connection.

3. Components are equal which means the network to how much subgroup is divide which equals 1 .

4. Diameter is equal 2 steps.

5. Clustering coefficient is 0.74 which is a very strong level of clustering. The highest eigenvector centrality in the sixth cluster was the Department of Biological Science (2).

\subsubsection{The Seventh Cluster}

The departments of the seventh cluster (community) are illustrated in Table 11. 
Table 11. Names of departments in the seventh cluster for the academic social network of KAU in RG

\begin{tabular}{|c|c|c|c|}
\hline Index & Name of department & Index & Name of department \\
\hline 1 & Computer Science & 33 & Medicine \\
\hline 2 & Biological & 35 & Economics \\
\hline 3 & Chemistry & 36 & Nuclear Engineering \\
\hline 4 & Biochemistry & 37 & Dentistry \\
\hline 5 & Mathematics & 46 & Education \\
\hline$\overline{6}$ & Electrical \& Computer Eng. & 48 & Management Information Sys. \\
\hline 7 & English Language Institute & 52 & Conservative Dental Sciences \\
\hline 8 & Information Systems & 55 & Arid Land Agriculture \\
\hline 9 & Physics & 57 & Medical Laboratories \\
\hline 10 & Information Technology & 60 & Marine Chemistry \\
\hline 11 & Industrial Engineering & 63 & Center of Excellence \\
\hline 12 & Psychology & 64 & Obstetrics and Gynecology \\
\hline 14 & European Languages & 65 & Computing \& Information Technology \\
\hline 16 & Statistics & 66 & Marketing \\
\hline 17 & Chemical \& Materials Eng. & 68 & Petroleum Geology \& Sedimentology \\
\hline$\overline{18}$ & Internal Medicine & 70 & Hematology \\
\hline 19 & Surgery & 76 & Anesthesia \\
\hline 20 & Food \& Nutrition & 84 & Special Education \\
\hline 21 & Information Science & 87 & Finance \\
\hline 22 & Mechanical Engineering & 97 & Center in Desalination Technology \\
\hline 25 & Preventive Dental Sciences & 107 & Structural Geology \& Remote Sensing \\
\hline 26 & Pediatrics & 112 & Earth Sciences \\
\hline 27 & Environmental Designs & 125 & Political Science \\
\hline 28 & Center of Excellence & 128 & Sciences \\
\hline 29 & Marine Biology & 135 & Travel \& Tourism Management \\
\hline 31 & Civil Engineering & & \\
\hline
\end{tabular}

\subsubsection{Basic Description}

1. The size of the seventh cluster is 50 departments.

2. Density is equal 0.75 which expresses a high level of connection.

3. Components are equal which means the network to how much subgroup is divide which equals1.

4. Diameter is equal 2 steps.

5. Clustering coefficient is 0.82 which is a very strong level of clustering. The highest eigenvector centrality in the seventh cluster was the Department of Biological Science (2).

\section{Comparing Random Models to Academic Social Networks}

The three most important models in the history of the social network are Erdos-Renyi Random Graph Model, Small-World Model and Scale-Free Model. Here, we compared between the degree distribution for Academic Social Network for KAU in RG and three models. The first model in the history of the social network, which was created by Paul Erdos and Alfred Renyi in the late of 1950s (Luke, 2015a). The degree distribution of academic social network is different from the degree distribution of random graph and scale-free models. However, it is slightly close to small world model (see Figure 7). 

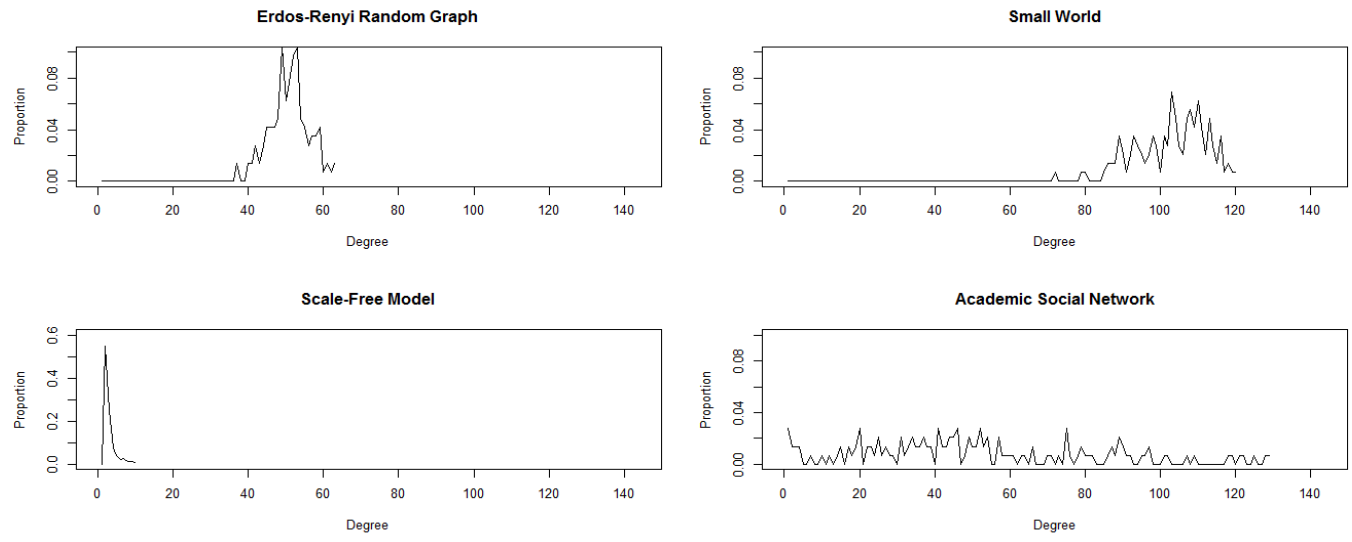

Figure 7. Comparing random models to the academic social network of KAU in RG

\section{Conclusions}

From the degree distribution in Figure 2, we noticed that the majority the departments have low degrees and the minority have high degrees. We noticed that Department of Chemistry (3) has had the highest degree for the academic social network of KAU in RG according Figure 1. From the vertex centrality with different types in Figure 3, Figure 4 and Figure 5, we found that Department of Electrical and Computer Engineering (6) have had the highest value in closeness and betweenness centrality, but in eigenvector, we found that the highest department was the Department of Chemistry (3). In fact, the eigenvector is the most expressive type of centrality because it represents the most central department which has a relationship with the most central departments as well. From the characteristics of the edges in Table 10 we found that the most two connected departments were the Department of Computer Science (1) and Department of Physics (9) through the edge with weight equal 248.

We have found seven clusters (communities) and there were four departments outside these clusters because they are isolated departments. In addition, the most related clusters by using clustering coefficient which is equal 1 the first and the fourth cluster. The degree distribution of the academic social network of KAU in RG in Figure 7 is different from the degree distribution of random graph and scale-free models. But this is slightly close to small world model. This paper has demonstrated the descriptive analysis of undirected academic social networks basic description, characteristics vertex and edges, sub- groups, and graph partitioning. The research paper has shed some light on the research characteristics and outlook within KAU different departments. It has demonstrated which department has had the highest and which department has had the lowest RG score at KAU in terms of inter-connectedness and isolation from other departments in the period from 10 November to 30 November 2017 when this research was conducted.

\section{Recommendations}

The study has many future recommendations which will be explained as in the following:

The departments at KAU can have more active participation in the academic social network such as RG, especially the isolated departments which are: 1) Home Economics (139), 2) Department of Emergency Medical Services (140), 4) Faculty of Medical Sciences (142) 4)and Faculty of Tourism (144). Creating a model for the social network of King Abdulaziz University in ResearchGate. This research can be applied on colleges instead of departments. The data can be collected for a period and used in time series analysis to observe the evolution of any university network. A comparison study of the communications between (and among) departments in RG and another academic social network such that Academia may result in a new understanding of researchers' communications' research impact and the effectiveness of such research platforms for researchers.

\section{Funding}

This project was funded by the Deanship of Scientific Research (DSR) at King Abdulaziz University, Jeddah, under grant number (G:1534-247-1440). The authors, therefore, acknowledge with thanks for DSR's technical and financial support. 


\section{References}

De Andrade, R. L., \& R^ego, L. C. (2018). The use of nodes attributes in social network analysis with anapplication to an international trade network. Physical A: Statistical Mechanics and its Applications, 491, 249-270.

Elsayed, A. M. (2016). The use of academic social networks among Arab researchers: A survey. Social Science Computer Review, 34(3), 378-391.

Hassan, S.-U., Imran, M., Gillani, U., Aljohani, N. R., Bowman, T. D., \& Didegah, F. (2017, Nov 01). Measuring social media activity of scientific literature: an exhaustive comparison of Scopus and novel Altmetrics big data. Scientometrics, 113(2), 1037-1057. https://doi.org/10.1007/s11192-017-2512-x

Hu, J., \& Zhang, Y. (2017). Discovering the interdisciplinary nature of big data research through social network analysis and visualization. Scientometrics, 112(1), 91-1091. https://doi.org/10.1007/s11192-017-2383-1

Kolaczyk, E. D., \& Cs'ardi, G. (2014). Descriptive analysis of network graph characteristics. In Statistical analysis of network data with $R$ (pp. 43-60). New York, NY: Springer New York. https://doi.org/10.1007/978-1-4939-0983-4

Kraker, P., \& Lex, E. (2015). A critical look at the ResearchGate score as a measure of scientific reputation. In Proceedings of the quantifying and analysing scholarly communication on the web workshop (ascw'15). Web science conference.

Luke, D. A. (2015a). The network analysis 'five-number summary'. In A user's guide to network analysis in $R$ (pp. 11-16). Cham: Springer International Publishing. https://doi.org/10.1007/978-3-319-23883-82

Luke, D. A. (2015b). Subgroups. In A user's guide to network analysis in $R$ (pp.105-123). Cham: Springer International Publishing. https://doi.org/10.1007/978-3-319-23883-8 8

Mislove, A., Marcon, M., Gummadi, K. P., Druschel, P., \& Bhattacharjee, B. (2007). Measurement and analysis of online social networks. In Proceedings of the 7th Acmsigcomm conference on internet measurement (pp. 29-42). New York, NY, USA: ACM. https://doi.org/10.1145/1298306.1298311

Mosaad, K. (2016). How will Saudi Arabia revamp its education system?. Fair Observer.

Pandia, M. K., \& Bihari, A. (2015). Important author analysis in research professionals' relationship network based on social network analysis metrics. In Computational Intelligence in Data Mining (Vol. 3, pp. 185-194). Springer, New Delhi.

Srivastava, A., \& Gupta, D. J. (2014, February). Social network analysis: Hardly easy. In 2014 International Conference on Reliability Optimization and Information Technology (ICROIT) (pp. 128-135). IEEE. https://doi.org/10.1109/ICROIT.2014.6798311

\section{Copyrights}

Copyright for this article is retained by the author(s), with first publication rights granted to the journal.

This is an open-access article distributed under the terms and conditions of the Creative Commons Attribution license (http://creativecommons.org/licenses/by/4.0/). 